\title{
Article \\ Disease Severity and Risk Factors of 30-Day Hospital Readmis- sion in Pediatric Hospitalizations for Pneumonia
}

\author{
Motomori O. Lewis ${ }^{1}$, Phuong T. Tran ${ }^{2}$, Yushi Huang ${ }^{3}$, Raj A. Desai ${ }^{4}$, Yun Shen ${ }^{5}$, and Joshua D. Brown ${ }^{6 *}$ \\ 1 Department of Pharmaceutical Outcomes and Policy, University of Florida College of Pharmacy, Gaines- \\ ville, Florida, 32610, USA; motomorilewis@ufl.edu \\ 2 Department of Pharmaceutical Outcomes and Policy, University of Florida College of Pharmacy, Gaines- \\ ville, Florida, 32610, USA; Faculty of Pharmacy, Ho Chi Minh City University of Technology (HUTECH), \\ Ho Chi Minh City, 700000, Vietnam; phuong.tran@ufl.edu \\ 3 Department of Pharmaceutical Outcomes and Policy, University of Florida College of Pharmacy, Gaines- \\ ville, Florida, 32610, USA; yushi.h@ufl.edu \\ 4 Department of Pharmaceutical Outcomes and Policy, University of Florida College of Pharmacy, Gaines- \\ ville, Florida, 32610, USA; raj.desai@ufl.edu \\ 5 Department of Pharmaceutical Outcomes and Policy, University of Florida College of Pharmacy, Gaines- \\ ville, Florida, 32610, USA; yunshen@ufl.edu \\ 6 Center for Drug Evaluation \& Safety and Department of Pharmaceutical Outcomes and Policy, University of \\ Florida College of Pharmacy, Gainesville, Florida, 32610, USA; joshua.brown@cop.ufl.edu \\ * Correspondence: joshua.brown@cop.ufl.edu; Tel.: +1-352-294-8593
}

\begin{abstract}
Pneumonia is the leading cause of hospitalization in pediatric patients. Disease severity greatly influences pneumonia progression and adverse health outcomes such as hospital readmission. Hospital readmissions have become a measure of healthcare quality to reduce excess expenditures. The aim of this study was to examine 30-day all-cause readmission rates and evaluate the association between pneumonia severity and readmission among pediatric pneumonia hospitalizations. Using 2018 Nationwide Readmissions Database (NRD), we conducted a cross-sectional study of pediatric hospitalizations for pneumonia. Pneumonia severity was defined by the presence of respiratory failure, sepsis, mechanical ventilation, dependence on long-term supplemental oxygen, and/or respiratory intubation. Outcomes of interest were 30-day all-cause readmission, length of stay, and cost. The rate of 30-day readmission for the total sample was $5.9 \%, 4.7 \%$ for non-severe pneumonia, and $8.7 \%$ for severe pneumonia $(\mathrm{p}<0.01)$. Among those who were readmitted, hospitalizations for severe pneumonia had a longer length of stay ( 6.5 vs. 5.4 days, $\mathrm{p}<0.01)$ and higher daily cost $(\$ 3,246$ vs. $\$ 2,679, p<0.01)$ than admissions for non-severe pneumonia. Factors associated with 30-day readmission were pneumonia severity, immunosuppressive conditions, length of stay, and hospital case volume. To reduce potentially preventable readmissions, clinical interventions to improve the disease course and hospital system interventions are necessary.
\end{abstract}

Keywords: pediatric pneumonia; hospital readmission; healthcare quality; hospital costs

\section{Introduction}

Approximately 8-30\% of all pediatric hospital admissions are potentially preventable [1-3], costing the healthcare system up to $\$ 561.6$ million in 2017 [1]. Since the passage of the Patient Protection and Affordable Care Act, hospital readmissions have become a measure of healthcare quality promoted by insurers and policymakers to reduce excess healthcare expenditures [4-6]. Hospitals failing to meet readmission benchmarks may be subject to reduced reimbursement from the Centers for Medicaid \& Medicare Services (CMS) $[5,6]$. For children's hospitals, which were exempt from reimbursement penalties, readmission remains a quality measure upheld by several state Medicaid agencies [2]. Based on the influence of these organizations, many children's hospitals have implemented practices to reduce readmissions and improve hospital care. Readmissions serve 
as an indicator of inadequate disease management during an initial visit. They also reflect the comprehensiveness of inpatient care including discharge processes, patient education, and follow-up care [7].

Amid national efforts to reduce hospital readmissions and unnecessary healthcare utilization, pneumonia is the single leading cause of hospitalization in pediatric patients [8]. Globally, pneumonia is among the top causes of death in children under 5 years [9]. In addition to its high morbidity and mortality, the economic burden of inpatient care for pneumonia is substantial at nearly $\$ 6.5$ billion [10]. Pneumonia can present with a host of complications. Factors that influence the severity of pneumonia include age, malnutrition, underlying chronic conditions, and timeliness and appropriateness of treatment [11-13]. Treatment of pneumonia is hindered by a lack of established criteria to define severity and standardized care plans that address complications [11]. Most children's hospitals rely on guideline recommendations to generate institution-specific process of care plans for complicated pneumonia. Disease severity plays a large role in pneumonia progression, especially regarding adverse health outcomes.

We hypothesize that the rate of hospital readmissions will be higher in patients with severe pneumonia. To our best knowledge, no prior studies have evaluated the association between disease severity and 30-day all-cause readmission in pediatric pneumonia patients. Additionally, despite many policies, hospital readmission rates did not improve from 2010-2016 [14], yet infection diagnostics, vaccine promotion, and medical care have advanced in recent years [15-18]. Therefore, the aim of this study was to examine pneumonia readmission rates by severity and evaluate the association between pneumonia severity and 30-day all-cause readmission in pediatric pneumonia hospitalizations using the 2018 Nationwide Readmissions Database (NRD).

\section{Materials and Methods}

We conducted a cross-sectional analysis using hospital discharge data from the 2018 Nationwide Readmissions Database (NRD) curated by Healthcare Cost and Utilization Project (HCUP), Agency for Healthcare Research and Quality (AHRQ). NRD is an allpayer database detailing inpatient admission records from U.S. community hospitals [19]. It includes about 17 million unweighted and 36 million weighted hospitalizations per year. Hospitalizations for any condition among all ages are included in the sample. NRD is a multi-level dataset in that it includes both discharge-level and hospital-level data. To facilitate national readmissions estimates and account for over- or under-sampling, discharge weights are applied through post-stratification on patient and hospital characteristics. Further, NRD allows tracking of individual patients with a unique patient linkage variable that serves as a patient identifier across hospitalizations within one state throughout a calendar year. As a result, individuals seeking care at different hospitals may be identified. The NRD sampling frame covers 27 geographically distributed states across the nation (a full list of HCUP data partners can be found here: https://www.hcupus.ahrq.gov/db/hcupdatapartners.jsp), representing the majority of all U.S. hospitalizations (56.6\%) and residents (57.8\%). NRD is part of the HCUP family of databases curated for healthcare researchers; the project is supported by the Agency for Healthcare Research and Quality. All databases within the collection are publicly available for purchase.

Our study sample consists of hospitalizations in a pediatric cohort under 18 years of age. To identify pneumonia cases, we selected all hospitalizations with a primary ICD9/10 diagnosis code for pneumonia (ICD codes shown in Supplement Table S1). To further identify eligible cases, we also selected cases with a primary diagnosis for either septicemia (038.xx) or acute respiratory failure (518.8x) and secondary diagnosis for pneumonia because these conditions are often related to pneumonia. Our pneumonia definition was developed from previously validated algorithms to identify pneumonia in administrative claims data [20-23]. Patients with missingness on key variables (length of stay, total charge, death, visit link, and days to event) are excluded. To qualify as an index admission eligible for readmissions analysis, patients must not have died in hospital during their 
visit and must have been discharged from the hospital before December 1, 2015, to provide a 30-day time frame for analyzing readmissions. In our readmissions sample, we excluded patients with an elective hospital admission. The sample selection flow diagram is shown in Figure 1.

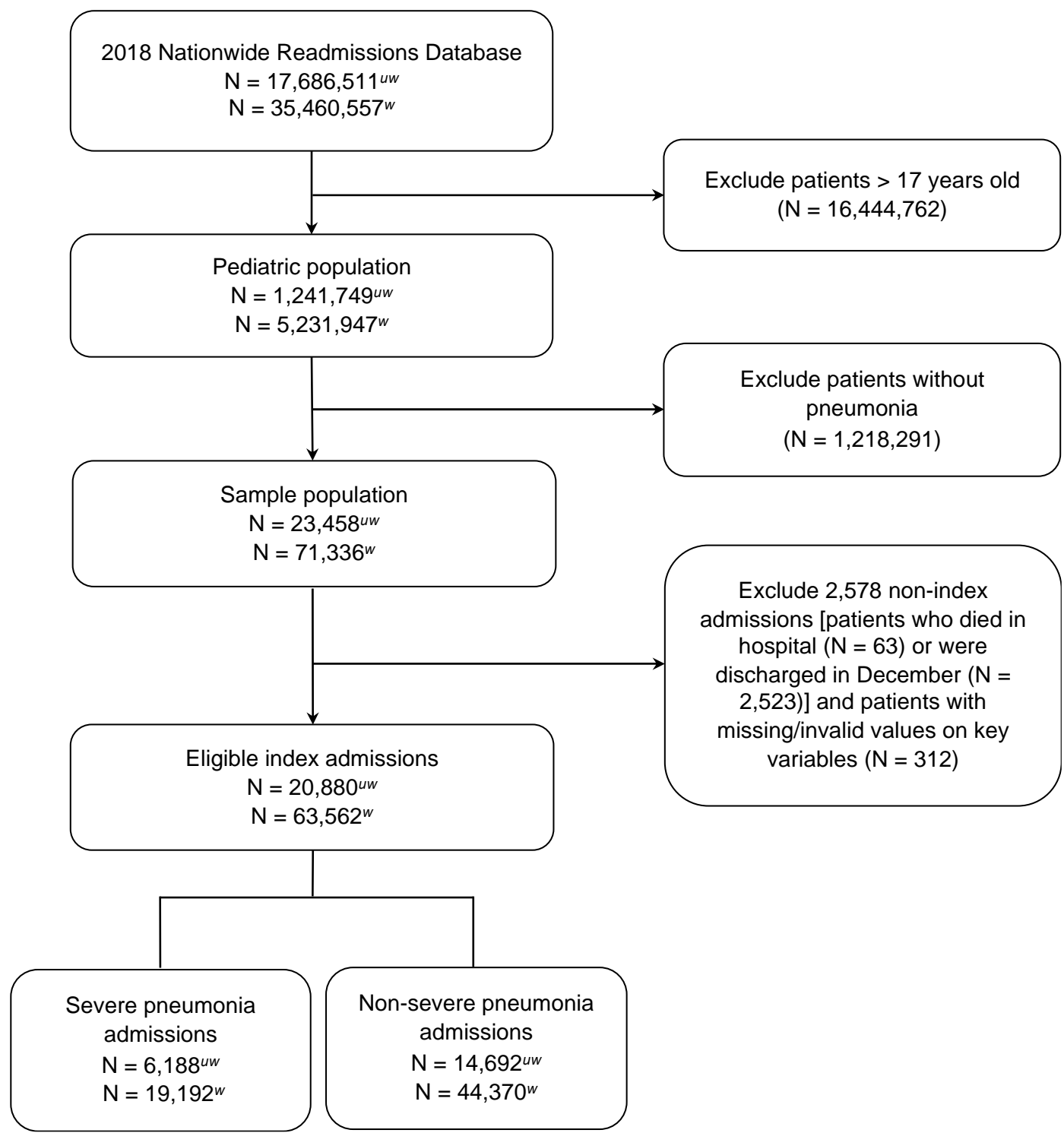

Figure 1. Flow diagram for study sample selection ${ }^{*} \mathrm{uw}=$ unweighted; $\mathrm{w}=$ weighted

After identifying qualifying hospitalizations, the sample was then stratified into severe and non-severe pneumonia cases. To assess pneumonia severity, we constructed a unique severity indicator developed from patient comorbidities and hospital procedures. We considered severe admissions to be those that included any of the following conditions: respiratory failure, sepsis, mechanical ventilation, dependence on long-term supplemental oxygen, and/or respiratory intubation [24,25]. We define the ICD-9/10-CM/PCS diagnostic and procedural codes for this definition in Table S1. Patient-level (clinical and demographic) and hospital-level characteristics were selected as potential risk factors for readmission. Using the HCUP Elixhauser Comorbidity Software [26], we calculated the Elixhauser mortality risk score, which is an index of comorbidity measures generated from diagnosis codes on the patient record [27]. We also added patient demographics (e.g., age, gender, income quartile, patient location, and state residency), relevant clinical comorbidities (e.g., asthma, acute bronchitis/bronchiolitis, cancer, chronic pulmonary disease, sickle cell disease, and cystic fibrosis; Table S1), and hospital information (e.g., 
teaching status, bed size, and urban-rural designation). In order to account for the variation in pneumonia cases at each hospital, hospital case volume was calculated by totaling the number of pneumonia cases for each hospital [28].

Our primary outcome of interest was 30-day all-cause hospital readmission rate. To examine the clinical and economic burden, we determined the length of stay, and daily and total hospitalization cost. Cost was calculated as the product of total charge and the hospital-specific cost-to-charge ratio provided by HCUP [29].

Descriptive and summary statistics were reported among the total sample and by pneumonia severity. We compared patient and hospitalization characteristics across the two groups to examine significant differences. For continuous variables, we conducted independent t-tests. For binary and multi-level categorial variables, we used Chi-Square and Fisher's exact tests, respectively. Associations between pneumonia severity and readmission were evaluated using logistic regression to estimate the odds ratio with $95 \%$ confidence intervals. Covariates that differed significantly across severity groups and identified as potential confounders were adjusted for in the model. To ensure the robustness of our findings, we performed additional sensitivity analyses: (1) we adjusted the outcome to include only pneumonia-specific readmissions and (2) we used a second measure of severity - the HCUP severity variable included in the NRD dataset. We applied an a priori significance level of $\alpha=0.05$. All analyses were conducted using SAS software 9.4, SAS Institute Inc., Cary, NC, USA.

\section{Results}

Our nationally representative study sample included a total of 20,880 hospitalizations for pediatric pneumonia (Figure 1). Of the sample, one-third (29.6\%) were classified as admissions for severe pneumonia (Table 1).

Table 1. Patient and hospital characteristics of pediatric pneumonia admissions, 2018

\begin{tabular}{|c|c|c|c|c|}
\hline Characteristics & $\begin{array}{c}\text { Total Sample } \\
\mathrm{N}=20,880(100 \%)\end{array}$ & $\begin{array}{c}\text { Non-severe } \\
\text { Pneumonia } \\
\mathrm{N}=14,692(70.4 \%)\end{array}$ & $\begin{array}{c}\text { Severe pneumonia } \\
\mathrm{N}=6,188(29.6 \%)\end{array}$ & P-value \\
\hline \multicolumn{5}{|c|}{ Patient Demographic Characteristics } \\
\hline Age group & & & & $<0.01$ \\
\hline $0-2$ years & $8610(41.2)$ & $5886(40.1)$ & $2724(44.0)$ & \\
\hline 3-7 years & $7109(34.1)$ & $5114(34.8)$ & $1995(32.2)$ & \\
\hline $8-12$ years & $3017(14.4)$ & $2176(14.8)$ & $841(13.6)$ & \\
\hline 13-17 years & $2144(10.3)$ & $1516(10.3)$ & $628(10.2)$ & \\
\hline Female & $9882(47.3)$ & $6956(47.4)$ & $2926(47.3)$ & 0.94 \\
\hline Income quartile & & & & $<0.01$ \\
\hline $0-25$ th percentile & $7271(34.8)$ & $5293(36.0)$ & $1978(32.0)$ & \\
\hline 26th-50th percentile (median) & $5955(28.5)$ & $4090(27.8)$ & $1865(30.1)$ & \\
\hline 51st-75th percentile & $4678(22.4)$ & $3267(22.24)$ & $1411(22.8)$ & \\
\hline 76th-100th percentile & $2976(14.3)$ & $2042(13.9)$ & $934(15.1)$ & \\
\hline Patient location & & & & $<0.01$ \\
\hline Large central metro & $5548(26.6)$ & $3905(26.6)$ & $1643(26.7)$ & \\
\hline Large fringe metro & $4519(21.6)$ & $3081(21.0)$ & $1438(23.2)$ & \\
\hline Medium metro & $4373(20.9)$ & $2964(20.2)$ & $1409(22.8)$ & \\
\hline Small metro & $2405(11.5)$ & $1768(12.0)$ & $637(10.3)$ & \\
\hline Micropolitan & $2276(10.9)$ & $1651(11.2)$ & $625(10.1)$ & \\
\hline Non-metro or micropolitan & $1759(8.4)$ & $1323(9.0)$ & $436(7.1)$ & \\
\hline
\end{tabular}




\begin{tabular}{|c|c|c|c|c|}
\hline In-state resident & $19883(95.2)$ & $14058(95.7)$ & $5825(94.1)$ & $<0.01$ \\
\hline Emergency department & $15559(74.5)$ & $10916(74.3)$ & $4644(75.1)$ & 0.25 \\
\hline Admitted on weekend & $5209(25.0)$ & $3650(24.8)$ & $1559(25.2)$ & 0.59 \\
\hline Season & & & & 0.19 \\
\hline Winter & $6292(30.1)$ & $4437(30.2)$ & $1855(30.0)$ & \\
\hline Spring & $6238(29.9)$ & $4337(29.5)$ & $1901(30.7)$ & \\
\hline Summer & $3084(14.8)$ & $2161(14.7)$ & $923(14.9)$ & \\
\hline Fall & $5266(25.2)$ & $3757(25.6)$ & $1509(24.4)$ & \\
\hline Expected primary payer & & & & $<0.01$ \\
\hline Medicare & $125(0.6)$ & $82(0.6)$ & $43(0.7)$ & \\
\hline Medicaid & $12567(60.2)$ & $8728(59.4)$ & $3839(62.0)$ & \\
\hline Private insurance & $7193(34.5)$ & $5141(35.0)$ & $2052(33.2)$ & \\
\hline Self-pay & $440(2.1)$ & $336(2.3)$ & $104(1.7)$ & \\
\hline No charge & $555(2.7)$ & $405(2.8)$ & $150(2.4)$ & \\
\hline \multicolumn{5}{|c|}{ Patient Clinical Characteristics } \\
\hline \multicolumn{5}{|l|}{ Respiratory conditions } \\
\hline Asthma & $7244(34.7)$ & $5001(34.0)$ & $2243(36.3)$ & $<0.01$ \\
\hline Acute bronchitis/ bronchiolitis & $2805(13.4)$ & $1579(10.8)$ & $1226(19.8)$ & $<0.01$ \\
\hline Chronic pulmonary disease & $7399(35.4)$ & $5111(34.8)$ & $2288(37.0)$ & $<0.01$ \\
\hline \multicolumn{5}{|l|}{$\begin{array}{l}\text { Immunosuppressive } \\
\text { conditions }\end{array}$} \\
\hline Cancer, any type & $187(0.9)$ & $134(0.9)$ & $53(0.9)$ & 0.70 \\
\hline Cystic fibrosis & $466(2.2)$ & $434(3.0)$ & $32(0.5)$ & $<0.01$ \\
\hline Sickle cell disease & $280(1.3)$ & $257(1.8)$ & $23(0.4)$ & $<0.01$ \\
\hline HCUP severity class & & & & $<0.01$ \\
\hline Minor & $6199(29.7)$ & $5556(37.8)$ & $643(10.4)$ & \\
\hline Moderate & $8199(39.3)$ & $6754(46.0)$ & $1445(23.4)$ & \\
\hline Major & $4177(20.0)$ & $1860(12.7)$ & $2317(37.4)$ & \\
\hline Extreme & $2305(11.0)$ & $522(3.6)$ & $1783(28.8)$ & \\
\hline \multicolumn{5}{|c|}{ Hospital Characteristics } \\
\hline Hospital size & & & & $<0.01$ \\
\hline Small & $3142(15.1)$ & $2318(15.8)$ & $824(13.3)$ & \\
\hline Medium & $4406(21.1)$ & $3413(23.2)$ & $993(16.1)$ & \\
\hline Large & $13332(63.9)$ & $8961(61.0)$ & $4371(70.6)$ & \\
\hline $\begin{array}{c}\text { Hospital urban-rural } \\
\text { designation }\end{array}$ & & & & $<0.01$ \\
\hline Large metropolitan & $11234(53.8)$ & $7636(52.0)$ & $3598(58.1)$ & \\
\hline Small metropolitan & $7352(35.2)$ & $5153(35.1)$ & $2199(35.5)$ & \\
\hline Micropolitan & $1609(7.7)$ & $1293(8.8)$ & $316(5.1)$ & \\
\hline Non-urban & $685(3.3)$ & $610(4.2)$ & $75(1.2)$ & \\
\hline Hospital teaching status & & & & $<0.01$ \\
\hline Metro non-teaching & $1790(8.6)$ & $1465(10.0)$ & $325(5.3)$ & \\
\hline Metro teaching & $16796(80.4)$ & $11324(77.0)$ & $5472(88.4)$ & \\
\hline Non-metro hospital & $22.94(11.0)$ & $1903(13.0)$ & $391(6.3)$ & \\
\hline
\end{tabular}




\begin{tabular}{|c|c|c|c|c|}
\hline Hospital case volume & & & & $<0.01$ \\
\hline 0 -25th percentile & $5347(25.6)$ & $4341(29.5)$ & $1006(16.3)$ & \\
\hline 26th-50th percentile (median) & $5154(24.7)$ & $3750(25.5)$ & $1404(22.7)$ & \\
\hline 51st-75th percentile & $5165(24.7)$ & $3567(24.3)$ & $1598(25.8)$ & \\
\hline 76th-100th percentile & $5214(25.0)$ & 3034 (20.7) & $2180(35.2)$ & \\
\hline
\end{tabular}

In the overall cohort, nearly $90 \%$ of hospitalized patients were age 12 years and under. Females comprised $47 \%$ of the study sample. The greatest proportion of patients resided in areas with the lowest income quartile $(34.8 \%)$ and large central metropolitan counties. Three-fourths (74.5\%) of admissions originated in the emergency department and $25.0 \%$ were admitted on a weekend. Majority of patients had an expected primary payer of Medicaid (60.2\%) followed by private insurance (34.5\%). Overall, we found a high proportion of respiratory comorbidities [asthma $(34.7 \%)$, acute bronchitis/bronchiolitis $(13.4 \%)$, and chronic pulmonary disease $(35.4 \%)]$. The prevalence of immunosuppressive conditions was low in our sample [cancer $(0.9 \%)$, cystic fibrosis $(2.2 \%)$, and sickle cell disease $(1.3 \%)$ ]. Most hospitalizations for pneumonia occurred in large $(63.9 \%)$, teaching hospitals $(80.4 \%)$ in metropolitan areas $(89.0 \%)$.

There were significant differences in the clinical and hospital characteristics between non-severe and severe pneumonia admissions. The prevalence of respiratory conditions was significantly higher in the severe pneumonia group than the non-severe group [asthma (36.3 vs. 34.0\%, p<0.01), acute bronchitis/bronchiolitis (19.8 vs. $10.8 \%, \mathrm{p}<0.01)$, and chronic pulmonary disease $(37.0$ vs. $34.8 \%, \mathrm{p}<0.01)]$. With the exception of cancer $(0.9 \%$ for both groups, $\mathrm{p}=0.70$ ), the frequency of admissions with immunosuppressive conditions was significantly lower among those with severe pneumonia compared to non-severe pneumonia [cystic fibrosis $(0.5$ vs. $3.0 \%, \mathrm{p}<0.01)$, and sickle cell disease $(0.4 \mathrm{vs} .1 .8 \%$, $\mathrm{p}<0.01)]$. Hospitals within the $76^{\text {th }}-100^{\text {th }}$ percentile of pneumonia case volume admitted a greater proportion of severe pneumonia cases than non-severe cases (35.2 vs. 20.7\%, $\mathrm{p}<0.01)$.

Readmissions and related clinical and economic burden

The rate of 30 -day all-cause readmissions was $5.9 \%$ for the total sample, $4.7 \%$ for nonsevere pneumonia and $8.7 \%$ for severe pneumonia $(\mathrm{p}<0.01$; Table 2 ). Of the total readmissions, $28.4 \%$ were specifically for pneumonia. Compared to the index admission, hospital stays for readmissions were generally longer and cost more per day. Among those who were readmitted, hospitalizations for severe pneumonia had a longer length of stay (6.5 vs. 5.4 days, $\mathrm{p}<0.01)$ and higher daily cost $(\$ 3,246$ vs. $\$ 2,679, \mathrm{p}<0.01)$ than admissions for non-severe pneumonia. The total economic burden for pediatric pneumonia hospital admissions costs over $\$ 205$ million, with severe pneumonia admissions resulting in the majority of costs (>\$111 million). Thirty-day readmissions for pneumonia contributed to an excess of \$22 million for hospitalization costs in 2018.

Table 2. Clinical and economic burden of pediatric pneumonia readmissions, 2018

\begin{tabular}{|c|c|c|c|c|}
\hline Outcome & $\begin{array}{c}\text { Total Sample } \\
\mathrm{N}=20,880(100 \%)\end{array}$ & $\begin{array}{c}\text { Non-severe pneumonia } \\
\mathrm{N}=14,692(70.4 \%)\end{array}$ & $\begin{array}{c}\text { Severe pneumonia } \\
\mathrm{N}=6,188(29.6 \%)\end{array}$ & P-value \\
\hline 30-day readmission, all-cause & $1225(5.9)$ & $687(4.7)$ & $538(8.7)$ & $<\mathbf{0 . 0 1}$ \\
\hline $\begin{array}{c}\text { 30-day readmission, } \\
\text { pneumonia-specific }\end{array}$ & $348(1.7)$ & $210(1.4)$ & $138(2.2)$ & $<\mathbf{0 . 0 1}$ \\
\hline \multicolumn{5}{|c|}{ Index hospitalization } \\
\hline $\begin{array}{c}\text { Length of stay (days), } \\
\text { mean } \pm \text { SD }\end{array}$ & $3.8(4.1)$ & $3.0(2.9)$ & $5.7(5.7)$ & $<\mathbf{0 . 0 1}$ \\
\hline Daily cost (USD), mean \pm SD & $2,341(1,823)$ & $2,120(1,363)$ & $2,865(2,533)$ & $<\mathbf{0 . 0 1}$ \\
\hline Sum of total costs (USD) & $205,400,992$ & $93,869,832$ & $111,531,160$ & $<\mathbf{0 . 0 1}$ \\
\hline
\end{tabular}




\begin{tabular}{|c|c|c|c|c|}
\hline \multicolumn{5}{|c|}{ 30-day readmission } \\
\hline $\begin{array}{c}\text { Length of stay (days), } \\
\text { mean } \pm \text { SD }\end{array}$ & $5.9(5.9)$ & $5.4(5.5)$ & $6.5(6.4)$ & $<\mathbf{0 . 0 1}$ \\
\hline Daily cost (USD), mean \pm SD & $2,928(1,963)$ & $2,679(1,978)$ & $3,246(1,898)$ & $<\mathbf{0 . 0 1}$ \\
\hline Sum of total costs (USD) & $22,036,400$ & $10,340,098$ & $11,696,302$ & $<\mathbf{0 . 0 1}$ \\
\hline
\end{tabular}

Eight of the 10 most common reasons for readmission were respiratory-related conditions such as pneumonia (23.8\%), respiratory failure, insufficiency, or arrest $(11.8 \%)$, acute bronchitis $(7.7 \%)$, asthma $(6.8 \%)$, influenza $(3.9 \%)$, upper respiratory infections $(2.9 \%)$, aspiration pneumonitis $(2.7 \%)$ and respiratory signs and symptoms (e.g., epistaxis, hemorrhage, cough, dyspnea, shortness of breath; $2.5 \%)$. Other reasons for readmission included epilepsy or convulsions (3.6\%) and septicemia (3.5\%) (Table 3).

Table 3. Top 10 causes of readmissions based on Clinical Classifications Software Refined

\begin{tabular}{|l|c|}
\hline \multicolumn{1}{|c|}{ Principal Diagnosis } & N (\%) \\
\hline Pneumonia (except that caused by tuberculosis) & $291(23.8)$ \\
\hline Respiratory failure, insufficiency, or arrest & $144(11.8)$ \\
\hline Acute bronchitis & $94(7.7)$ \\
\hline Asthma & $83(6.8)$ \\
\hline Influenza & $48(3.9)$ \\
\hline Epilepsy or convulsions & $44(3.6)$ \\
\hline Septicemia & $43(3.5)$ \\
\hline Other specified upper respiratory infections & $36(2.9)$ \\
\hline Aspiration pneumonitis & $33(2.7)$ \\
\hline $\begin{array}{l}\text { Respiratory signs and symptoms (e.g., epistaxis, hemorrhage, cough, } \\
\text { dyspnea, shortness of breath, etc.) }\end{array}$ & $30(2.5)$ \\
\hline
\end{tabular}

\section{Risk factors for readmission}

Hospital admissions for severe pneumonia were associated with $44 \%$ increased odds of all-cause 30-day readmission compared to non-severe pneumonia admissions (OR 1.44, 95\% CI 1.26-1.65) (Table 4). Higher odds of readmission were also seen among teenagers (OR 1.40, 95\% CI 1.16-1.68) and children insured through Medicare (OR 2.17, 95\% CI 1.203.95) or Medicaid (1.20, 95\% CI 1.05-1.37). Patients with comorbidities such as cancer (OR $4.82,95 \%$ CI 3.43-6.77) or sickle cell disease (OR 2.14, 95\% CI 1.45-3.16) and admissions at metropolitan teaching hospitals (OR 1.48, 95\% CI 1.12-1.97) or hospitals with a higher pneumonia case volume $\left(26^{\text {th }}-50^{\text {th }}\right.$ percentile: OR $1.33,95 \%$ CI $1.08-1.64 ; 51^{\text {st }}-75^{\text {th }}$ percentile: OR $1.40,95 \%$ CI $1.13-1.74 ; 76^{\text {th }}-100^{\text {th }}$ percentile: OR $1.50,95 \%$ CI $\left.1.22-1.86\right)$ were also at increased odds of readmission. Admissions with an increased length of stay (OR 1.04, 95\% CI 1.03-1.06) and daily cost $\left(51^{\text {st }-75^{\text {th }}}\right.$ percentile: OR $1.29,95 \%$ CI $1.07-1.55 ; 76^{\text {th }}-100^{\text {th }}$ percentile: OR 1.40, 95\% CI 1.16-1.69) were indicative of greater odds of readmission. Those less likely to be readmitted were self-insured patients (OR 0.44, 95\% CI 0.22-0.86) and patients diagnosed with asthma (OR 0.78, 95\% CI 0.68-0.90).

Table 4. Risk factors associated with 30-day all-cause readmission

\begin{tabular}{|c|c|}
\hline Covariate & Adjusted Odds Ratio (95\% CI) \\
\hline Severe pneumonia & $1.44(1.26,1.65)^{*}$ \\
\hline
\end{tabular}




\begin{tabular}{|c|c|}
\hline Age group & \\
\hline $0-2$ years & Reference \\
\hline 3-7 years & $0.89(0.77,1.03)$ \\
\hline $8-12$ years & $1.02(0.85,1.22)$ \\
\hline $13-17$ years & $1.40(1.16,1.68)^{*}$ \\
\hline Female & $0.99(0.88,1.12)$ \\
\hline \multicolumn{2}{|l|}{ Expected primary payer } \\
\hline Private insurance & Reference \\
\hline Medicare & $2.17(1.20,3.95)^{*}$ \\
\hline Medicaid & $1.20(1.05,1.37)^{*}$ \\
\hline Self-pay & $0.44(0.22,0.86)^{*}$ \\
\hline No charge & $1.40(0.98,1.98)$ \\
\hline Length of stay & $1.04(1.03,1.06)^{*}$ \\
\hline \multicolumn{2}{|l|}{ Daily cost } \\
\hline 0-25th percentile & Reference \\
\hline 26th-50th percentile (median) & $1.15(0.95,1.38)$ \\
\hline 51st-75th percentile & $1.29(1.07,1.55)^{*}$ \\
\hline 76th-100th percentile & $1.40(1.16,1.69)^{*}$ \\
\hline Asthma & $0.78(0.68,0.90)^{*}$ \\
\hline Cancer, any type & $4.82(3.43,6.77)^{*}$ \\
\hline Cystic fibrosis & $0.89(0.62,1.27)$ \\
\hline Sickle cell disease & $2.14(1.45,3.16)^{*}$ \\
\hline \multicolumn{2}{|l|}{ Hospital size } \\
\hline Small & Reference \\
\hline Medium & $1.00(0.81,1.24)$ \\
\hline Large & $1.13(0.95,1.35)$ \\
\hline \multicolumn{2}{|l|}{ Hospital teaching status } \\
\hline Non-metropolitan hospital & Reference \\
\hline Metropolitan non-teaching & $0.84(0.57,1.23)$ \\
\hline Metropolitan teaching & $1.48(1.12,1.97)^{*}$ \\
\hline \multicolumn{2}{|l|}{ Hospital case volume } \\
\hline 0-25th percentile & Reference \\
\hline 26th-50th percentile (median) & $1.33(1.08,1.64)^{*}$ \\
\hline 51st-75th percentile & $1.40(1.13,1.74)^{*}$ \\
\hline 76th-100th percentile & $1.50(1.22,1.86)^{*}$ \\
\hline
\end{tabular}

${ }^{*}$ Odds ratio is statistically significant

Both sensitivity analyses were consistent with our main analysis (Tables S2, S3). When restricting the outcome to pneumonia-specific readmissions, only pneumonia severity (OR 1.48, 95\% CI 1.16-1.86), female sex (OR 1.27, 95\% CI 1.03-1.58), and hospitals with higher case volume were statistically significant for increased odds of readmission (51 $1^{\text {st }} 75^{\text {th }}$ percentile: OR $1.61,95 \%$ CI $1.09-2.38 ; 76^{\text {th }}-100^{\text {th }}$ percentile: OR $2.03,95 \%$ CI 1.38 2.99). Applying the HCUP severity class resulted in greater odds of readmission among severe pneumonia cases than our primary severity definition (moderate loss of function: OR 1.76, 95\% CI 1.43-2.15; major loss of function: OR 3.93, 95\% CI 3.19-4.85; extreme loss 
of function: OR 5.53, 95\% CI 4.35-7.04), and significantly reduced odds of readmission among patients with cystic fibrosis (OR 0.66, 95\% CI 0.47-0.93).

\section{Discussion}

Our nationally representative, cross-sectional study is among the first to demonstrate that pneumonia severity is associated with 30-day all-cause readmission in pediatric pneumonia hospitalizations. Patient demographics, such as teen age and being publicly insured, were non-modifiable risk factors for readmission. Patients with immunosuppressive conditions such as cancer and sickle cell disease were more likely to be readmitted than those with other clinical comorbidities, despite these diagnoses being rare and more prevalent among non-severe pneumonia cases. In addition, longer length of stay during an index hospitalization contributed to greater risk of readmission. Finally, we found that hospital case volume and teaching status were more predictive of readmission risk than hospital size.

Our sample had a 30 -day all-cause readmission rate of $5.9 \%$. These results corroborate previous literature on the sparse $(<10 \%)$ readmission prevalence for pediatric pneumonia [30-32]. Hospital readmission was more likely among patients with severe pneumonia. Despite conflicting evidence on whether disease severity is associated with readmission [33], there is a need to account for severity in future prediction models for pneumonia readmission. Risk stratification is a useful method to identify patients with more complications for treatment interventions and reduce the likelihood of rehospitalization [34]. Special attention is needed for children with immunosuppressive conditions. It is well established that readmission rates for pediatric pneumonia are higher among patients with chronic conditions [35-38]. However, in our sample, the presence of immunosuppressive diseases, such as cancer and SCD, showed an increased risk of readmission while chronic respiratory conditions such as asthma did not. While readmission in SCD and cancer may be attributable to other factors due to their underlying disease, pneumonia diagnosis presents an added layer of complexity and can lead to prolonged illness and complications. Vaccination can be considered to reduce pneumonia in this high-risk subgroup [37].

Our analysis demonstrated an association between longer length of stay and risk of readmission, as supported by a similar study of children with complicated pneumonia [39]. Contrary to these findings, other observational studies of pediatric readmissions in U.S. children's hospitals found no meaningful association between index hospitalization length of stay and risk of readmission in children's hospitals [40,41]. Additionally, increasing length of stay to reduce readmission was not a practical, cost-effective solution for most diagnoses [40]. While there is still conflict on whether index admission length of stay is a valuable measure for predicting readmissions, length of stay may be useful as an indicator of hospital efficiency and quality-of-care. Guideline-concordant therapy has been associated with shorter length of stay in adults hospitalized with community-acquired pneumonia [42]. Future research is needed to explore whether a guideline-directed treatment approach can be extended to the pediatric population and reduce resource utilization for pneumonia.

Several hospital-level factors were significant predictors of all-cause readmission. In particular, admissions occurring at hospitals with higher pneumonia case volume and metropolitan teaching status were more likely to result in readmission. Previous literature found hospital-level factors contributed to nearly $80 \%$ of preventable readmissions [43]. Given the significance of hospital variation in readmissions, a systems-based approach may be necessary to prevent readmissions and improve hospital quality. Evidence suggests that discharge transition plans, follow-up care, and addressing adverse social determinants of health are useful interventions to reduce hospital readmission rates [44,45].

Our study has noteworthy strengths. We used a nationally representative sample of admissions from community hospitals within the U.S., providing generalizability to the entire U.S. pediatric population. Further, NRD data is curated from statewide 
partnerships, permitting the linkage of patients hospitalized in more than one institution across the state. However, there are also a few limitations to our investigation. First, we were not able to identify admissions that were transferred to the intensive care unit (ICU) due to the lack of availability of UB-04 revenue codes in our data source. ICU admission is an established indicator of pneumonia severity [25]. Second, our data does not include other relevant clinical information such as respiratory laboratory values, interventions, and medication use. Third, while we were able to track the order of patients' hospitalizations within the year, our cross-sectional study design is not suitable to determine the timing of events within a hospitalization.

\section{Conclusions}

Our study examined the clinical and economic burden of pediatric pneumonia readmissions. Hospitalizations for pediatric pneumonia promote a significant economic burden, especially for severe pneumonia. We found that pneumonia severity is associated with risk of readmission. Risk factors for 30-day all-cause readmission also include immunosuppressive conditions, index admission length of stay, and hospital case volume. Both clinical and hospital systems-level interventions are necessary to reduce potentially preventable readmissions.

Supplementary Materials: The following supporting information can be downloaded at: www.mdpi.com/xxx/s1, Table S1: ICD-10 diagnosis and procedure codes for pneumonia and relevant comorbidities; Table S2: Sensitivity analysis 1 - Risk factors associated with 30-day pneumoniaspecific readmission for pediatric pneumonia; Table S3: Sensitivity analysis 2 - Risk factors associated with 30-day all-cause readmission for pediatric pneumonia using HCUP severity class.

Author Contributions: Conceptualization, J.D.B.; methodology, M.O.L., P.T.T., R.A.D., Y.H., and Y.S., J.D.B.; formal analysis, M.O.L.; data resources, J.D.B.; writing-original draft preparation, M.O.L., P.T.T., R.A.D., Y.H.; writing - review and editing, M.O.L., P.T.T., R.A.D., Y.H., Y.S., and J.D.B.; visualization, M.O.L. and P.T.T.; supervision, J.D.B. All authors have read and agreed to the published version of the manuscript.

Funding: This research received no external funding.

Institutional Review Board Statement: The study was conducted in accordance with the Declaration of Helsinki and approved by the University of Florida Institutional Review Board (IRB201900471; July 15, 2020).

Informed Consent Statement: Patient consent was waived due to the public nature of the data resource and minimal risk to study participants.

Data Availability Statement: Data are used under a Data Use Agreement that does not allow distribution of source data. HCUP's Nationwide and State-Specific Databases are available for purchase at: https://www.distributor.hcup-us.ahrq.gov/. Programming codes are available upon request.

Conflicts of Interest: The authors declare no conflict of interest. 


\section{References}

1. McDermott KW, Jiang HJ. Characteristics and Costs of Potentially Preventable Inpatient Stays, 2017: Statistical Brief \#259. Healthcare Cost and Utilization Project (HCUP) Statistical Briefs. Agency for Healthcare Research and Quality (US); 2006.

2. Toomey SL, Peltz A, Loren S, et al. Potentially Preventable 30-Day Hospital Readmissions at a Children's Hospital. Pediatrics. 2016;138(2). doi:10.1542/peds.2015-4182

3. Payne NR, Flood A. Preventing Pediatric Readmissions: Which Ones and How? The Journal of Pediatrics. 2015;166(3):519-520. doi:10.1016/j.jpeds.2014.12.020

4. Fontanarosa PB, McNutt RA. Revisiting Hospital Readmissions. JAMA. 2013;309(4):398-400. doi:10.1001/jama.2013.42

5. Joynt KE, Jha AK. Thirty-Day Readmissions - Truth and Consequences. New England Journal of Medicine. 2012;366(15):13661369. doi:10.1056/NEJMp1201598

6. Kocher RP, Adashi EY. Hospital Readmissions and the Affordable Care Act: Paying for Coordinated Quality Care. JAMA. 2011;306(16):1794-1795. doi:10.1001/jama.2011.1561

7. AHRQ. Pediatric 30-Day Lower Respiratory Infection Readmission Measure. Agency for Healthcare Research and Quality, Rockville, MD. https://www.ahrq.gov/pqmp/measures/pediatric-respiratory-infection-readmissions.html

8. Witt WP, Weiss AJ, Elixhauser A. Overview of Hospital Stays for Children in the United States, 2012: Statistical Brief \#187. Healthcare Cost and Utilization Project (HCUP) Statistical Briefs. Agency for Healthcare Research and Quality (US); 2006.

9. Black RE, Cousens S, Johnson HL, et al. Global, regional, and national causes of child mortality in 2008: a systematic analysis. The Lancet. 2010;375(9730):1969-1987. doi:10.1016/S0140-6736(10)60549-1

10. Liang L, Moore B, Soni A. National Inpatient Hospital Costs: The Most Expensive Conditions by Payer, 2017: Statistical Brief \#261. Healthcare Cost and Utilization Project (HCUP) Statistical Briefs. Agency for Healthcare Research and Quality (US); 2006.

11. Dean P, Florin TA. Factors Associated With Pneumonia Severity in Children: A Systematic Review. J Pediatric Infect Dis Soc. 2018;7(4):323-334. doi:10.1093/jpids/piy046

12. Williams DJ, Zhu Y, Grijalva CG, et al. Predicting Severe Pneumonia Outcomes in Children. Pediatrics. 2016;138(4):e20161019. doi:10.1542/peds.2016-1019

13. de Benedictis FM, Kerem E, Chang AB, Colin AA, Zar HJ, Bush A. Complicated pneumonia in children. The Lancet. 2020/09/12/ 2020;396(10253):786-798. doi:https://doi.org/10.1016/S0140-6736(20)31550-6

14. Auger KA, Harris JM, Gay JC, et al. Progress (?) Toward Reducing Pediatric Readmissions. Journal of Hospital Medicine. 2019;14(10):618-621. doi:10.12788/jhm.3210

15. Zar HJ, Andronikou S, Nicol MP. Advances in the diagnosis of pneumonia in children. BMJ (Clinical research ed). 2017;358:j2739. doi:10.1136/bmj.j2739

16. Gereige RS, Laufer PM. Pneumonia. Pediatrics In Review. 2013;34(10):438-456. doi:10.1542/pir.34.10.438

17. Jain S, Williams DJ, Arnold SR, et al. Community-Acquired Pneumonia Requiring Hospitalization among U.S. Children. New England Journal of Medicine. 2015;372(9):835-845. doi:10.1056/NEJMoa1405870

18. Griffin MR, Zhu Y, Moore MR, Whitney CG, Grijalva CG. U.S. Hospitalizations for Pneumonia after a Decade of Pneumococcal Vaccination. New England Journal of Medicine. 2013;369(2):155-163. doi:10.1056/NEJMoa1209165

19. HCUP Nationwide Readmissions Database (NRD). Healthcare Cost and Utilization Project (HCUP). https://www.hcupus.ahrq.gov/nrdoverview.jsp

20. Williams DJ, Shah SS, Myers A, et al. Identifying Pediatric Community-Acquired Pneumonia Hospitalizations: Accuracy of Administrative Billing Codes. JAMA Pediatrics. 2013;167(9):851-858. doi:10.1001/jamapediatrics.2013.186

21. Barber C, Lacaille D, Fortin PR. Systematic Review of Validation Studies of the Use of Administrative Data to Identify Serious Infections. Arthritis Care \& Research. 2013;65(8):1343-1357. doi:10.1002/acr.21959

22. Whittle J, Fine MJ, Joyce DZ, et al. Community-Acquired Pneumonia: Can It Be Defined with Claims Data? American Journal of Medical Quality. 1997;12(4):187-193. doi:10.1177/0885713x9701200404

23. Olasupo O, Xiao H, Brown JD. Relative Clinical and Cost Burden of Community-Acquired Pneumonia Hospitalizations in Older Adults in the United States - A Cross-Sectional Analysis. Vaccines. 2018;6(3):59.

24. Florin TA, Brokamp C, Mantyla R, et al. Validation of the Pediatric Infectious Diseases Society-Infectious Diseases Society of America Severity Criteria in Children With Community-Acquired Pneumonia. Clinical Infectious Diseases. 2018;67(1):112-119. doi:10.1093/cid/ciy031

25. Harris M, Clark J, Coote N, et al. British Thoracic Society guidelines for the management of community acquired pneumonia in children: update 2011. Thorax. 2011;66(Suppl 2):ii1. doi:10.1136/thoraxjnl-2011-200598

26. HCUP Elixhauser Comorbidity Software Refined for ICD-10-CM, v2021.1. Agency for Healthcare Research and Quality, Rockville, MD. https://www.hcup-us.ahrq.gov/toolssoftware/comorbidityicd10/comorbidity icd10.jsp

27. Elixhauser A, Steiner C, Harris DR, Coffey RM. Comorbidity Measures for Use with Administrative Data. Medical Care. 1998;36(1):8-27.

28. O'Brien SM, DeLong ER, Peterson ED. Impact of Case Volume on Hospital Performance Assessment. Archives of Internal Medicine. 2008;168(12):1277-1284. doi:10.1001/archinte.168.12.1277

29. HCUP Cost-to-Charge Ratio (CCR) for the Nationwide Readmissions Database (NRD). Agency for Healthcare Research and Quality, Rockville, MD. https://www.hcup-us.ahrq.gov/db/ccr/ip-ccr/ip-ccr.jsp

30. Neuman MI, Hall M, Gay JC, et al. Readmissions among children previously hospitalized with pneumonia. Pediatrics. 2014;134(1):100-109. doi:10.1542/peds.2014-0331 
31. Faverio P, Compagnoni MM, Della Zoppa M, et al. Rehospitalization for pneumonia after first pneumonia admission: Incidence and predictors in a population-based cohort study. PLOS ONE. 2020;15(6):e0235468. doi:10.1371/journal.pone.0235468

32. Berry JG, Toomey SL, Zaslavsky AM, et al. Pediatric Readmission Prevalence and Variability Across Hospitals. JAMA. 2013;309(4):372-380. doi:10.1001/jama.2012.188351

33. Shorr AF, Zilberberg MD, Reichley R, et al. Readmission Following Hospitalization for Pneumonia: The Impact of Pneumonia Type and Its Implication for Hospitals. Clinical Infectious Diseases. 2013;57(3):362-367. doi:10.1093/cid/cit254

34. Weinreich M, Nguyen OK, Wang D, et al. Predicting the Risk of Readmission in Pneumonia. A Systematic Review of Model Performance. Annals of the American Thoracic Society. 2016;13(9):1607-1614. doi:10.1513/annalsats.201602-135sr

35. Leyenaar JK, Lagu T, Shieh M-S, Pekow PS, Lindenauer PK. Management and outcomes of pneumonia among children with complex chronic conditions. Pediatr Infect Dis J. 2014;33(9):907-911. doi:10.1097/INF.0000000000000317

36. Nakamura MM, Zaslavsky AM, Toomey SL, et al. Pediatric Readmissions After Hospitalizations for Lower Respiratory Infections. Pediatrics. 2017;140(2). doi:10.1542/peds.2016-0938

37. Wang L, Feng Z, Shuai J, Liu J, Li G. Risk factors of 90-day rehospitalization following discharge of pediatric patients hospitalized with mycoplasma Pneumoniae pneumonia. BMC Infectious Diseases. 2019/11/12 2019;19(1):966. doi:10.1186/s12879-0194616-9

38. Ambroggio L, Herman H, Fain E, Huang G, Florin TA. Clinical Risk Factors for Revisits for Children With Community-Acquired Pneumonia. Hospital Pediatrics. 2018;8(11):718-723. doi:10.1542/hpeds.2018-0014

39. Gross CJ, Porter JJ, Lipsett SC, Monuteaux MC, Hirsch AW, Neuman MI. Variation in Management and Outcomes of Children With Complicated Pneumonia. Hospital Pediatrics. 2021;11(3):207-214. doi:10.1542/hpeds.2020-001800

40. Gay JC, Hall M, Markham JL, Bettenhausen JL, Doupnik SK, Berry JG. Association of Extending Hospital Length of Stay With Reduced Pediatric Hospital Readmissions. JAMA Pediatrics. 2019;173(2):186-188. doi:10.1001/jamapediatrics.2018.3815

41. Brogan TV, Hall M, Williams DJ, et al. Variability in Processes of Care and Outcomes Among Children Hospitalized With Community-acquired Pneumonia. Pediatr Infect Dis J. 2012;31(10):1036-1041. doi:10.1097/INF.0b013e31825f2b10

42. McCabe C, Kirchner C, Zhang H, Daley J, Fisman DN. Guideline-Concordant Therapy and Reduced Mortality and Length of Stay in Adults With Community-Acquired Pneumonia: Playing by the Rules. Archives of Internal Medicine. 2009;169(16):15251531. doi:10.1001/archinternmed.2009.259

43. Brittan M, Shah SS, Auger KA. Preventing Pediatric Readmissions: How Does the Hospital Fit In? Pediatrics. 2016;138(2). doi:10.1542/peds.2016-1643

44. deJong NA, Kimple KS, Morreale MC, Hang S, Davis D, Steiner MJ. A Quality Improvement Intervention Bundle to Reduce 30Day Pediatric Readmissions. Pediatr Qual Saf. 2020;5(2):e264-e264. doi:10.1097/pq9.0000000000000264

45. Nakamura MM, Toomey SL, Zaslavsky AM, et al. Measuring Pediatric Hospital Readmission Rates to Drive Quality Improvement. Academic Pediatrics. 2014/09/01/ 2014;14(5, Supplement):S39-S46. doi:https://doi.org/10.1016/j.acap.2014.06.012 